\title{
Gemella morbillorum endocarditis of pulmonary valve:a case report
}

\author{
Dan $\mathrm{Li}^{1 \dagger}$, Zhicheng Zhu' ${ }^{1}$, Xiaomei Zheng ${ }^{1}$, Weitie Wang ${ }^{2 \dagger}$, Rihao $\mathrm{Xu}^{1}$ and Kexiang Liu ${ }^{1 *}$
}

\begin{abstract}
Background: Pulmonary valve infective endocarditis is a rare finding for endocarditis. Infective endocarditis caused by Gemella morbillorum remains a scanty occurrence.

Case presentation: This is a case reported of a 28-year-old Chinese male with endocarditis caused by pulmonary valve infection of Gemella morbillorum associated with congenital ventricular septal defect, atrial septal defect and double-chambered right ventricle. The patient presented with fever, shortness of breath, progressively worsening exertional fatigue, dyspnea and weight loss for 3 months. The diagnosis was made with transthoracic echocardiogram, blood cultures, and post-operative pathology. The patient developed congestive heart failure and was managed with aggressive antibiotic therapy followed by surgery. He underwent replacement of the pulmonary valve with an aortic bioprosthetic valve, repair of ventricular septal defect and atrial septal defect, reconstruction of the right ventricular outlflow tract, and excision of vegetations. His postoperative recovery was uneventful. No bacteria were isolated from the excised tissues. He was asymptomatic without recurrence at 3-month follow-up.

Conclusions: The rare pathogen such as Gemella morbillorum can be the cause of infective endocarditis and timely surgical repair is necessary if the infection is refractory or there is progression of congestive heart failure under antibiotic cover.
\end{abstract}

Keywords: Infective endocarditis, Gemella morbillorum, Atrial septal defect, Ventricular septal defect, Double-chambered right ventricle

\section{Background}

G. morbillorum was originally described by Tunnicliff in 1917 [1]. The organism was relocated to the genus Gemella in 1988 [2]. Because of its characteristics of facultative anaerobic organism, G. morbillorum usually behaves as a commensal organism of the mucous membranes and is a part of the normal flora of human oropharynx, upper respiratory, gastrointestinal system, and genitourinary system [3]. It has been implicated as a causative pathogen in central nervous system infection, arthritis, pneumonia, pleural empyema, Ludwig's angina, mediastinitis and osteomyelitis, hepatic abscess, peritonitis, and cardiovascular system infection.

\footnotetext{
* Correspondence: kxliu64@hotmail.com

${ }^{\dagger}$ Equal contributors

'Department of Cardiovascular Surgery, The Second Hospital of Jilin University, 218 Ziqiang Street, Nanguan District, Changchun, Jilin 130041, People's Republic of China

Full list of author information is available at the end of the article
}

Although G. morbillorum possesses very low virulence comparing to some other pathogens, it was reported to cause endocarditis [4-7]. The morbidity of G. morbillorum endocarditis remains a rare occurrence so far. Both native and prosthetic valves can be affected by G. morbillorum. Mitral and aortic valves were more commonly infected compared to tricuspid and pulmonary valve. Mitral and aortic valves are affected in almost equal number of cases, while the pulmonary valve is rarely involved. Pulmonary valve infective endocarditis (IE) caused by G. morbillorum is an extremely rare occurrence and only one case has been reported in the literature hitherto [8]. This is the 1st case reported of pulmonary valve IE caused by G. morbillorum in a young Chinese man who had a congenital ventricular septal defect (VSD), atrial septal defect (ASD) and double-chambered right ventricle (DCRV).

\section{Case presentation}

A 28-year-old Chinese man was admitted to our hospital because of fever, chills and rigors, asthenia, malaise, 
cough, shortness of breath, edema and wasting for three days. His past medical history included an admission to the hospital 3 months ago due to bilateral pneumonia. At that time, the patient had intermittent, moderate grade fever, associated with chills and rigors. The fever was temporarily relieved with antipyretics. Laboratory studies showed white blood cells $12220 / \mathrm{mm}^{3}$ (82\% neutrophils). Chest computed tomography demonstrated multiple patches high density in the upper right lung, multiple nodular high density in the lower right lung, a thick-walled cavity in the lower left lung, several swollen soft tissue shadows in the mediastinum, and an enlarged spleen. A transthoracic echocardiogram (TTE) showed VSD, ASD, DCRV with anomaly hypertrophied muscle bands in right ventricular outlflow tract (RVOT), continuous-wave Doppler study demonstrated a peak systolic gradient of $72 \mathrm{mmHg}$ across the RVOT, trace tricuspid valve regurgitation, the thickened pulmonary valve and left ventricular ejection fraction (EF) of $57 \%$. The diagnoses of pneumonia, congenital heart disease of VSD, ASD and DCRV were made. He was treated with cefminox sodium $2 \mathrm{~g}$ iv q12 $\mathrm{h}$. He was afebrile in $72 \mathrm{~h}$, and 14 days later he was discharged home in good condition. After discharge, the patient experienced intermittent, moderate fever and was treated with oral antibiotics. He had been complaining of edemas of lower extremities, wasting and $15-\mathrm{kg}$ weight loss of 3 months' duration.

On admission, the patient was febrile $\left(38.3{ }^{\circ} \mathrm{C}\right)$, tachypnoic, anxious, and tachycardic with a heart rate of $110 \mathrm{bpm}$. His blood pressure was 120/80 $\mathrm{mmHg}$. The patient was fully conscious with normal neurological examination. Auscultation of the chest was clear bilaterally without any adventitious sounds. There was a systolic murmur at the left sternal border and a diastolic murmur in the pulmonary valve area. An electrocardiogram revealed sinus tachycardia. Laboratory studies showed the following pathological values: hemoglobin $91 \mathrm{~g} / \mathrm{L}$, white blood cells $7100 / \mathrm{mm}^{3}$ (82\% neutrophils), platelet count $88000 / \mathrm{mm} 3$, albumin $28.9 \mathrm{~g} / \mathrm{L}$.

A repeat TTE (Fig. 1) revealed a vegetation of $18 \times$ $4 \mathrm{~mm}$ on the pulmonary valve with moderate pulmonary valve regurgitation, VSD, ASD and DCRV, and the other valves were unremarkable. Normal size of left ventricle was noted with an left ventricular EF of 54\%. Three sets of blood cultures (each set contained one aerobic and one anaerobic vial) were obtained over a period of $24 \mathrm{~h}$. Intravenous ceftriaxone and diuretics were initiated. After 2 days of treatment he became afebrile. On the third day of admission, all the three cultures grew alphahemolytic, catalase-negative Gram positive cocci. Susceptibility testing of the organism was done using the disc diffusion method. On the seventh day of admission, all of the three collected blood cultures (6 vials) grew $G$. morbillorum. The organism isolated was highly sensitive to penicillin, ceftriaxone, rifampicin, vancomycin and ciprofloxacin and therefore the above regimen was continued. On the eighth day of admission the patient's clinical condition got worse with aggravated exertional fatigue and dyspnea, and TTE demonstrated severe pulmonary valve regurgitation. In light of these findings and the patient's worsening clinical manifestations, a decision was made for urgent surgical treatment.

The surgical approach was via median sternotomy. After systemic heparinization, cardiopulmonary bypass (CPB) was instituted via ascending aortic and bicaval cannulation. After aortic cross-clamping, cardiac arrest was achieved through antegrade administration of cold blood cardioplegic solution. The perimembranous VSD $(0.5 \times 0.4 \mathrm{~cm})$, ASD $(0.5 \times 0.6 \mathrm{~cm})$ were repaired via an oblique right atriotomy. Tricuspid valve was normal, while left and right valve leaflets of the pulmonary valves were severely destructed with a large vegetation on the anterior leaflet of pulmonary valve observed. The leaflets of pulmonary valve and vegetation were excised (Fig. 2), and DCRV was corrected through pulmonary valve and tricuspid valve followed by fixation of aortic bioprosthetic valve into the pulmonic valve annulus. Anomalous septal and parietal muscle bundles seen in RVOT divided the right ventricle into a proximal high-pressure chamber and a distal low-pressure chamber, and they were carefully resected during surgery. The main pulmonary artery was then widened with a pericardial patch. The patient was rewarmed and successfully weaned from $\mathrm{CPB}$.

The patient's recovery was uneventful, while his temperature increased abruptly to $39.5{ }^{\circ} \mathrm{C}$ on the postoperative day 4. Laboratory studies showed white blood cells $20200 / \mathrm{mm}^{3}$ (89.7\% neutrophils). Blood cultures with three separate blood specimens were obtained. In addition to previous medications, vancomycin $2 \times 1 \mathrm{~g} /$ day IV was added. He became afebrile in 2 days with the combination of antibiotics. Vancomycin was discontinued in 1 weeks, while ceftrixone was continued. Subsequent blood cultures showed negative. Culture of the excised tissue specimen was also negative for bacteria. The patient received 4 weeks antibiotic treatment and recovered well after surgery. He was discharged in good condition and remained asymptomatic at his 3-month follow-up.

\section{Discussion}

G. morbillorum was originally isolated by Tunnicliff in 1917. Infections caused by G. morbillorum are rare, and G. morbillorum endocarditis is a rare clinical entity. About 45 cases of G. morbillorum endocarditis were reported in the literature hitherto. 

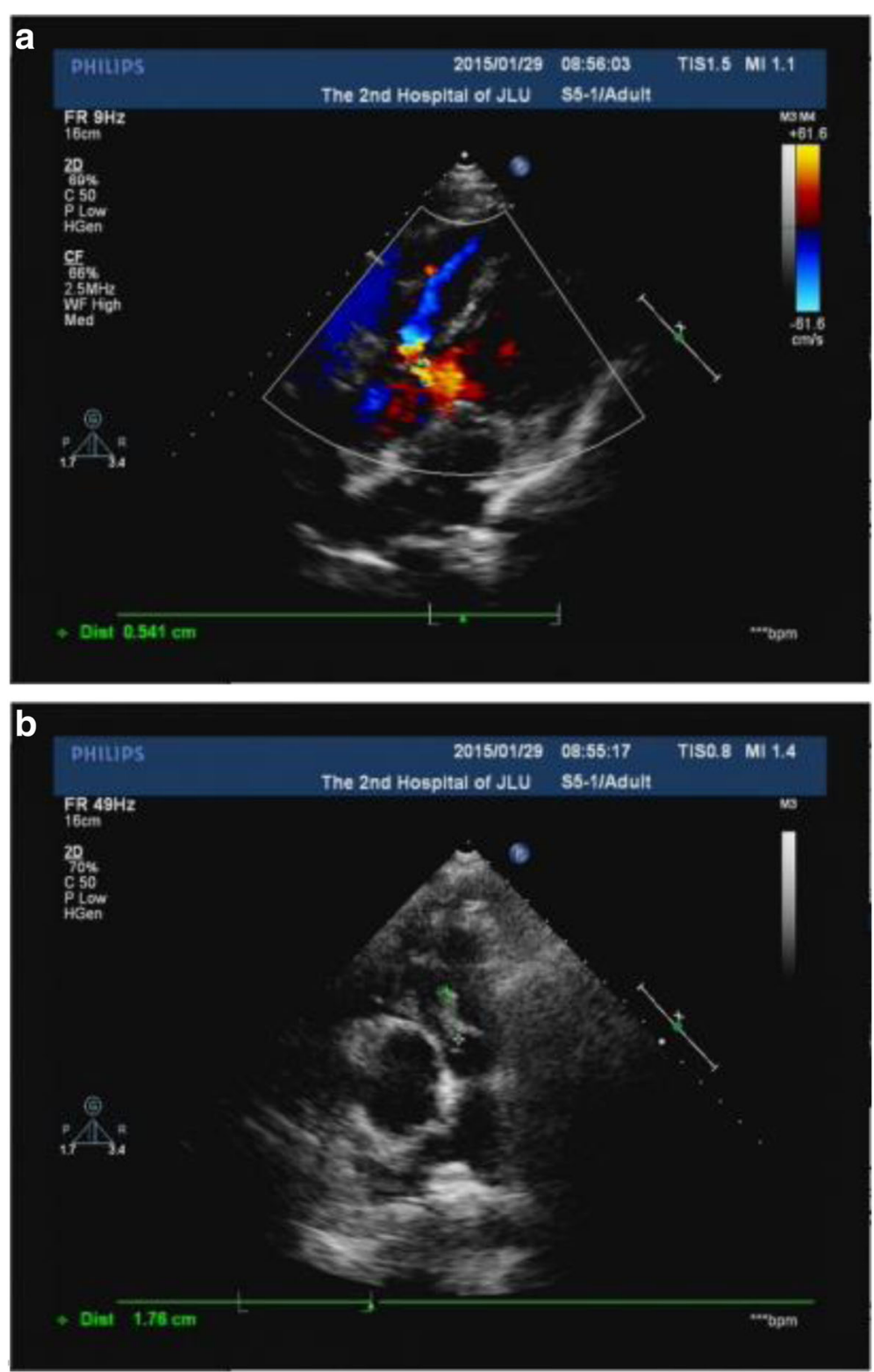

Fig. 1 a Transthoracic parasternal short axis view showing vegetation (white arrow) on pulmonary valve. b Transthoracic echocardiography showing a small perimembranous type VSD with left to right shunt

Predisposing factors for G. morbillorum endocarditis include underlying cardiac pathologies, such as preexisting valvular lesions and congenital heart diseases, etc. Any of the valves can be affected, including native valve and prosthetic valve. It has been reported that mitral and aortic valves were affected in an almost equal number of cases, sometimes even simultaneously; while the tricuspid valve and pulmonary valve is only rarely involved.

Our patient met the Duke's criteria [9] for IE. The major criteria met included positive echocardiographic evidence of vegetations, worsening pulmonary valvular regurgitation, and positive blood culture. The minor criteria met by this patient included fever, the predisposing heart condition of a congenital VSD, ASD and DCRV. The definitive source of infection for the organism was not found. Pulmonary valve IE is a rare finding accounting for less than $2 \%$ of hospital admissions for endocarditis [10]. The majority of pulmonary valve IE cases $(55 \%)$ occurred in patients with congenital heart disease. The occurrence of pulmonary valve IE caused by G. morbillorum is extremely rare. Only one case of pulmonary valve IE caused by G. morbillorum in a case of ASD was reported in the literature so far [8]. ASD 


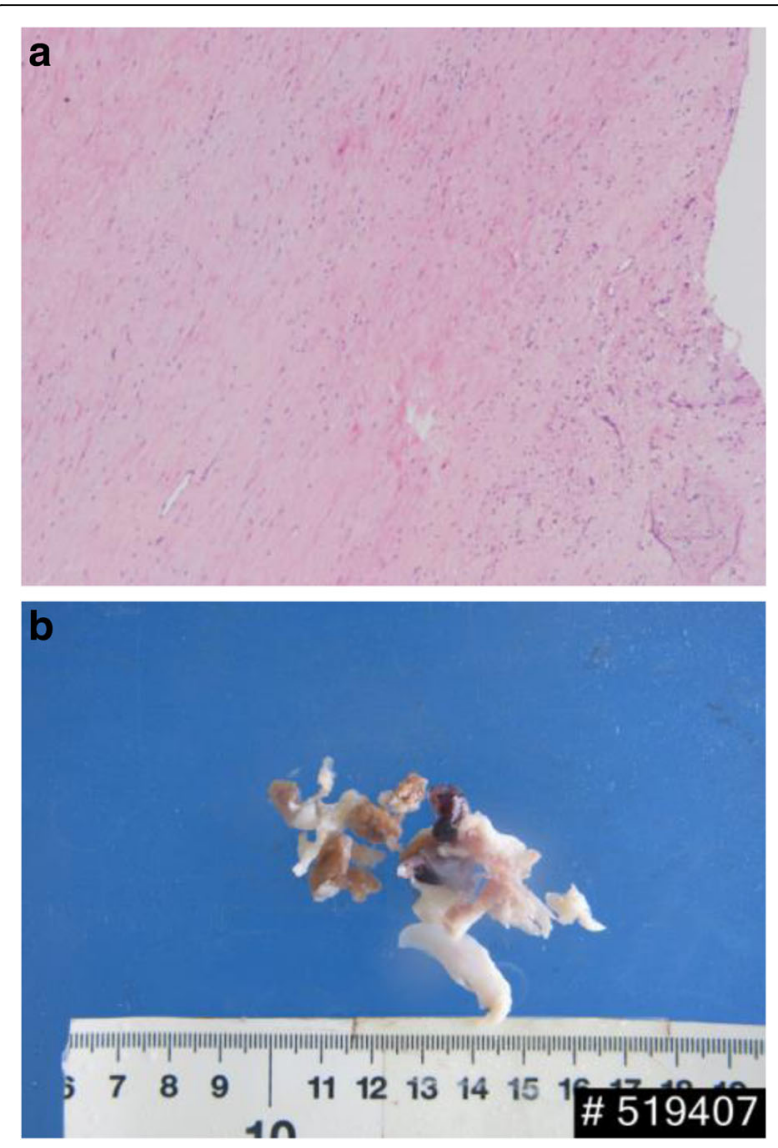

Fig. 2 a The resected left and right valve leaflets of the pulmonary valves were severely destructed with a large vegetation on the anterior leaflet of the pulmonary valve. $\mathbf{b}$ Pathologic specimen of the valve tissue (pulmonary valve) showed fibrosis and myxoid degeneration

can be a cause of the pulmonary valve endocarditis which is subjected to damage due to increased blood flow although it is not included in the list of predisposing factors for G. morbillorum endocarditis. VSD is a risk factor of IE involving right heart structures. Birkenkamp et al. speculated that the high pressure flow created by the left-to-right shunt across the VSD damaged the pulmonary valve, making the valve more susceptible to bacterial infection [11]. Our patient suffered from pulmonary valve IE caused by G. morbillorum with ASD, VSD and DCRV. Blood cultures were taken when he was admitted to our hospital. After specimens of blood for culture were drawn, he was treated with susceptible antibiotics. After 2 days of treatment he became afebrile. But on the eighth day of admission the patient's conditions deteriorated with worsening pulmonary valve regurgitation and secondary heart failure which were difficult to be controlled by the medical treatment, we referred him for urgent surgical intervention to excise vegetations and infected tissue thoroughly, correct all cardiac defects and replace the pulmonary valve. The patient continued his antibiotics therapy after operation to control the recurrent of infection. But his temperature increased abruptly and the count of white blood cells also increased on the post-operative day 4 , another sensitive antibiotics vancomycin was added. He became afebrile with the combination of antibiotics. To prevent the recurrence of the infection, the patient received 4 weeks of antibiotics coverage. He recovered well and was discharged from the hospital.

\section{Conclusions}

The patients of G. morbillorum endocarditis of pulmonary valve can be treated effectively with sensitive antibiotics. While the patient presents abrupt worsening and refractory heart failure, one of the key factors for the patient to recovery is urgent surgical repair of all cardiac defects, excision of vegetations and infected tissue.

\section{Abbreviations \\ ASD: Atrial septal defect; CPB: Cardiopulmonary bypass; DCRV: Double- chambered right ventricle; EF: Ejection fraction; G. morbillorum: Gemella morbillorum; IE: Infective endocarditis; RVOT: Right ventricular outlflow tract; TTE: Transthoracic echocardiogram; VSD: Ventricular septal defect}

\section{Acknowledgements}

We thank Dr. Liyan Zhuang and Dr. Liang Li who provided professional writing services.

\section{Funding}

This study received no external funding and was completely supported by the budget of the Department of Cardiovascular Surgery, The Second Hospital of Jilin University.

\section{Availability of data and materials}

All data supporting the conclusions of this article are included in this published article.

\section{Authors' contributions}

KXL designed the study, performed the operation, and revised the manuscript critically. $\mathrm{DL}$ was principally involved in data collection and manuscript writing. The other three authors $(Z C Z, X M Z, R H X)$ also have made substantial contributions in data interpretation. WTW was in charge of uploading the article and data interpretation. All authors read and approved the final manuscript. Both authors Dr. Dan Li and Weitie Wang who contributed equally in the study.

\section{Competing interests}

The authors declare that they have no competing interest.

\section{Consent for publication}

Written informed consent for publication of his clinical details and clinical images was obtained from the patient.

\section{Ethics approval and consent to participate}

The study design was approved from the Second Hospital of Jilin University Hospital Institutional Review Board (IRB number: 2016-104).

\section{Publisher's Note}

Springer Nature remains neutral with regard to jurisdictional claims in published maps and institutional affiliations.

\section{Author details}

${ }^{1}$ Department of Cardiovascular Surgery, The Second Hospital of Jilin University, 218 Ziqiang Street, Nanguan District, Changchun, Jilin 130041, People's Republic of China. ${ }^{2}$ Department of Cardiovascular Surgery, The First 
Hospital of Tianjin, No. 186 Nankou Road, Tianjin 300232, People's Republic of China.

Received: 15 August 2016 Accepted: 7 March 2017

Published online: 23 March 2017

\section{References}

1. Tunnicliff R. The cultivation of a micrococcus from blood in pre- and eruptive stages of measles. JAMA. 1917;68:1028-30

2. Kilpper-Balz R, Schleifer KH. Transfer of Streptococcus morbillorum to the genus Gemella as Gemella morbillorum comb. nov. Int J Syst Bacteriol. 1988;38:442-43.

3. Kofteridis DP, Anastasopoulos T, Panagiotakis S, Kontopodis E, Samonis G. Endocarditis caused by Gemella morbillorum resistant to $\beta$-lactams and aminoglycosides. Scand J Infect Dis. 2006:38:1125-27.

4. Al-Hujailan G, Lagacé-Wiens P. Mechanical valve endocarditis caused by Gemella morbillorum. J Med Microbiol. 2007;56:1689-91.

5. Kolhari VB, Kumar W, Agrawal N, Prakash SS. Gemella morbillorum endocarditis in hypertrophic cardiomyopathy: a rare organism causing a large vegetation and abscess in an uncommon setting. BMJ Case Rep. 2014 doi:10.1136/bcr-2014-203951.

6. Shahani L. Gemella morbillorum prosthetic aortic valve endocarditis. BM Case Rep. 2014. doi:10.1136/bcr-2014-207304.

7. Constantinos M, Marios S. Gemella morbillorum tricuspid valve endocarditis resulting in septic pulmonary emboli in a patient with intracranial hemorrhage. Int J Cardiol. 2015:184:769-71.

8. Agrawal N, Kariyappa M, Kolhari VB, Manjunath CN. Cauliflower-like deformation of pulmonary valve in a case of infective endocarditis by a rare organism: Gemella morbillorum. BMJ Case Rep. 2014. doi:10.1136/bcr2014-204726.

9. Durack DT, Lukes AS, Bright DK. New criteria for diagnosis of infective endocarditis: utilization of specific echocardiographic findings. Duke endocarditis Service. Am J Med. 1994;96:200-9.

10. Revilla A, López J, Villacorta E, Gómez I, Sevilla T, del Pozo MA, de la Fuente L, Manzano Mdel C, Mota P, Flórez S, Vilacosta I, Sarriá C, Sánchez M, San Román JA. Isolated right-sided valvular endocarditis in non-intravenous drug users. Rev Esp Cardiol. 2008:61:1253-59.

11. Birkenkamp KE, Jin JJ, Shivashankar R, Jouni H, Baddour LM, Blauwet LA. Ventricular septal defect and bivalvular endocarditis. Avicenna J Med. 2015;5:21-3.

\section{Submit your next manuscript to BioMed Central and we will help you at every step:}

- We accept pre-submission inquiries

- Our selector tool helps you to find the most relevant journal

- We provide round the clock customer support

- Convenient online submission

- Thorough peer review

- Inclusion in PubMed and all major indexing services

- Maximum visibility for your research

Submit your manuscript at www biomedcentral.com/submit 